\title{
The Influence of Place on Weight Gain during Early Childhood: A Population-Based, Longitudinal Study
}

Megan Ann Carter, Lise Dubois, Mark S. Tremblay, and Monica Taljaard

\begin{abstract}
The objective of this paper was to determine the influence of place factors on weight gain in a contemporary cohort of children while also adjusting for early life and individual/family social factors. Participants from the Québec Longitudinal Study of Child Development comprised the sample for analysis $(n=1,580)$. A mixed-effects regression analysis was conducted to determine the longitudinal relationship between these place factors and standardized BMI, from age 4 to 10 years. The average relationship with time was found to be quadratic (rate of weight gain increased over time). Neighborhood material deprivation was found to be positively related to weight gain. Social deprivation, social disorder, and living in a medium density area were inversely related, while no association was found for social cohesion. Early life factors and genetic proxies appeared to be important in explaining weight gain in this sample. This study suggests that residential environments may play a role in childhood weight change; however, pathways are likely to be complex and interacting and perhaps not as important as early life factors and genetic proxies. Further work is required to clarify these relationships.
\end{abstract}

KEYWORDS Children, Neighborhood, Residential characteristics, Environment, Body weight, Body mass index, Longitudinal study, Mixed-models, Social factors

\section{INTRODUCTION}

Childhood overweight and obesity have risen dramatically in the last 25 years in Canada ${ }^{1,2}$ and in many other countries. ${ }^{3,4}$ In 2004, $26 \%$ of Canadian children aged 2-17 years were overweight and $8 \%$ were obese. ${ }^{2}$ From 1978/1979 to 2004, the prevalence of overweight and obesity increased twofold among 6-17-year-olds. Due to this striking increase, as well as its potential for adversely affecting health both in the short and long term, being at excess weight during childhood has become a major global public health concern.

The etiologic literature on childhood weight status has tended to focus on individual characteristics rather than on broader contextual circumstances. The high prevalence of childhood obesity has not abated, suggesting that prevention

Carter and Dubois are with the Institute of Population Health, University of Ottawa, Ottawa, ON, Canada; Tremblay is with the Healthy Active Living and Obesity Research (HALO) CHEO Research Institute, Ottawa, ON, Canada; Taljaard is with the Ottawa Hospital Research Institute, Ottawa Hospital, Civic Campus, Ottawa, ON, Canada.

Correspondence: Megan Ann Carter, Institute of Population Health, University of Ottawa, Ottawa, ON, Canada. (E-mail: carter.megan@gmail.com) 
strategies, traditionally implemented at the individual level, may not be effective. Conceptualizing childhood obesity within multiple levels of influence, specifically within residential communities and over the life course, is necessary to design effective prevention strategies that shift the distribution of risk downward. This is consistent with a social-ecological theory of health. ${ }^{5}$

Neighborhoods are a natural way to conceptualize "place" in terms of child health and acquiring health resources. In the context of obesity, complex interactions between individuals, families, local communities, and institutions, as well as the broader social environment, lead neighborhoods to become geographical areas that can: (1) structure opportunities/barriers for children to be physically active and to eat healthy ${ }^{6}$ and (2) give rise to negative perceptions, which themselves may elicit chronic stress responses leading to weight gain. ${ }^{7}$

Studies are starting to find significant relationships between different neighborhood characteristics and weight status; ${ }^{8}$ however, this is a fairly new area of research where the literature is heterogeneous and mostly cross-sectional. ${ }^{9}$ To better make the case for causation, longitudinal studies are needed that use measured heights and weights. Accounting for early life factors known to be related to childhood obesity development, as well as individual and family-level social factors and measures of the family environment can provide a more holistic picture of why and how weight status changes over time in young children.

Among the few longitudinal studies investigating the influence of place on childhood weight status, findings include significant negative relationships between change in BMI and area greenness/degree of vegetation, ${ }^{10}$ neighborhood income/ deprivation, ${ }^{11}$ and perceived safety. ${ }^{12}$

Using the Québec Longitudinal Study of Child Development (QLSCD), the main objective of this study was to assess the influence of place factors on change in cohort children's standardized weight for height while also accounting for other potentially important early life and individual-/family-level explanatory factors. The overall hypothesis was that unfavorable neighborhood characteristics such as high material and social deprivation, high social disorder, and low population density would be positively related to weight gain, while favorable characteristics such as high social cohesion would be inversely related to weight gain in children.

\section{METHODS}

\section{Sample}

The QLSCD is a government-based cohort study conducted by the Institut de la Statistique du Québec (ISQ) to identify factors in early childhood that affect the health, social adjustment, and academic performance of young Quebeckers. ${ }^{13}$ The cohort is comprised of a representative sample of 2,120 children born in Québec in 1997/1998, who have been followed from 5 months of age.

Cohort children were randomly selected based on a three-stage, stratified design. ${ }^{14}$ The territory covered by the survey was first divided into primary sampling units (PSUs) based on broad regions of Québec. The PSUs were then divided into two groups: remote or nonremote. In stage 1, two out of the four remote PSUs were chosen, and all 11 nonremote PSUs were chosen. The second stage involved dividing the selected regions into secondary sampling units (SSUs) based on one or two county regional municipalities. These were further divided into two groups: areas registering a high number of births in 1996 and those registering a 
low number of births. A fixed number of SSUs were randomly selected from the low birthrate group, and all SSUs were selected from the high birthrate group. Finally, in the third stage, a fixed number of children were randomly selected from the selected SSUs, based on the 1997/1998 Québec Birth Registry. ${ }^{14}$ Sampling occurred throughout the year to minimize the potential impact of seasonal influences (see Figure 1 for the sampling process). Twins, children with major diseases at birth, and those living in Northern Québec, Cree or Inuit territory, or Indian reserves were excluded.

From 5 months to 8 years of age, data collection occurred annually (timing changed slightly when children began school). In order to minimize respondent burden, this changed to a biannual basis from 8 years onward. Data from 5 months to 10 years are used in the present analysis. Computer-assisted personal interviewing of the mother, in the child's home, was the primary method of data collection. ${ }^{13}$

\section{VARIABLES}

\section{Outcome}

The primary outcome for this study was weight relative to height, as measured by BMI $\left(\mathrm{kg} / \mathrm{m}^{2}\right)$, standardized for age and sex using the Centers for Disease Control and Prevention Growth Charts, to obtain BMI Z-scores. ${ }^{15}$ The Z-score is the deviation of the value for an individual from the mean value of the reference population divided by the standard deviation for the reference population; in this case, the reference population has been derived from five different US surveys. ${ }^{16}$ The use of the BMI Z-score has been recommended as superior than percentiles for use in longitudinal analyses. ${ }^{17}$

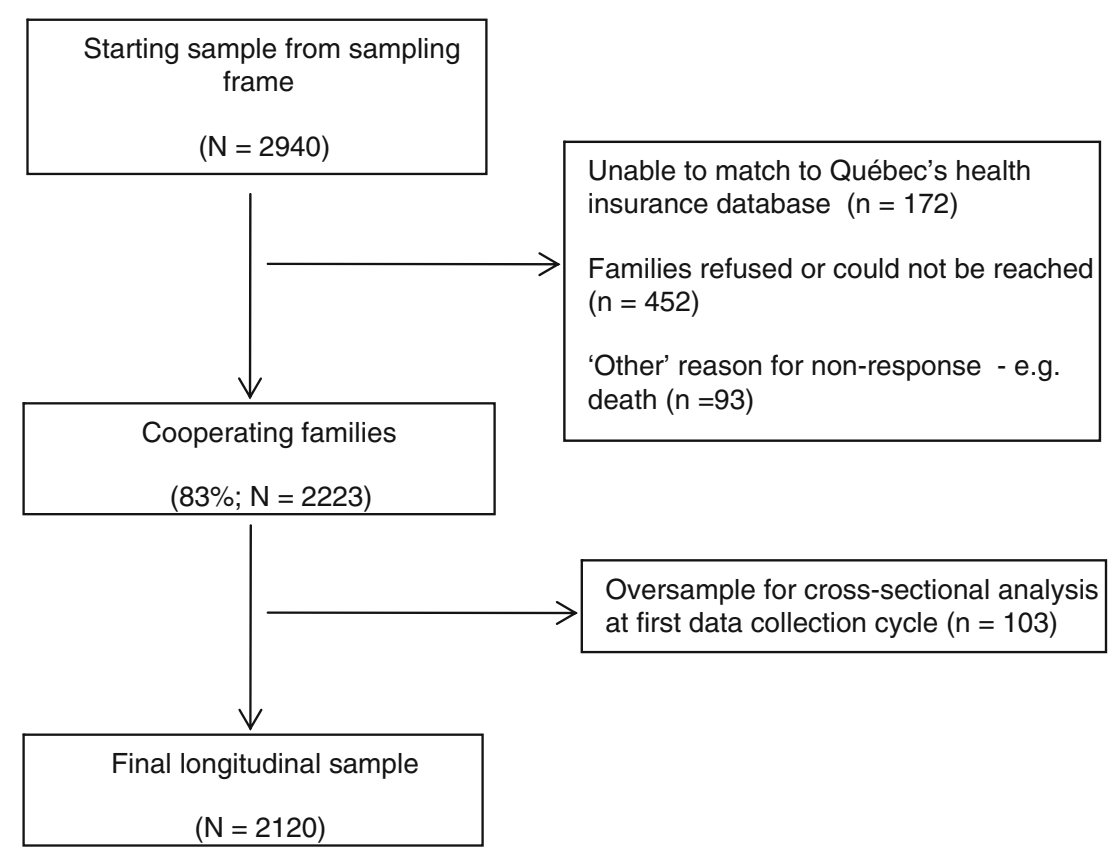

FIGURE 1. Sampling of children in the Québec Longitudinal Study of Child Development. 
Heights and weights were measured in the child's home by trained interviewers at the approximate ages of 4, 6, 7, 8, and 10 years. ${ }^{18}$ At each data collection cycle, body weight was measured in kilograms on scales set back to zero for each measurement. Children wore light clothing and no shoes. Height was measured in meters.

\section{Main Exposures-Place Factors}

Deprivation Two forms of deprivation, material and social, were measured by an area-based index developed by Pampalon and Raymond, ${ }^{19}$ based largely on the work of Peter Townsend. The index was derived by linking postal codes of participants at data collection cycle 1, when children were approximately 5 months of age, to census data (1996) describing enumeration areas. Principal components analysis was used to create the index using six socioeconomic indicators: proportion of persons who have no high-school diploma; ratio of employment to total population; average income; proportion of persons who are separated, divorced, or widowed; proportion of people living alone; and proportion of single-parent families. The first three indicators form the material dimension of the index. This refers to the general inability of area residents to obtain the goods and conveniences that are a part of everyday life. The latter three indicators form the social dimension of the index, which refers to the fragmentation and weakening of the household structure. The index has been previously used to assess disparities in Canadian mortality rates $^{20}$ and by the Québec Government to assess community service needs. ${ }^{21}$

For ease of interpretation and consistent with another paper, both dimensions were divided into population quintiles, from quintile 1 (least disadvantaged) to 5 (most disadvantaged), and then dichotomized into "deprived" (quintiles 4 and 5) versus "not deprived" (quintiles $1-3$ ). ${ }^{22}$

Perceived Neighborhood Social Cohesion and Disorder Neighborhood social cohesion and disorder were measured by two scales that have been adapted from the work of Barnes-McGuire ${ }^{23}$ and the Canadian National Longitudinal Survey of Children and Youth. ${ }^{24}$ Both are based on the mother's perception of her neighborhood. Items forming the social cohesion scale assess the level of agreement to five statements about the support of neighbors, while items on the social disorder scale assess the presence and severity of four types of problems in the neighborhood. ${ }^{25}$ The items for each of the scales can be found in Appendix 1. Scale scores were calculated by averaging item responses for each scale. Social cohesion scores range from 1 to 4 , where higher scores indicate a less cohesive neighborhood. Social disorder scores range from 1 to 3 with a lower score indicating the presence of social problems. Both scales were dichotomized to increase interpretability. Social cohesion was dichotomized based on the 50th percentile. For social disorder, children were categorized as either having a perfect score of 3 (no social problems at all in the neighborhood) versus $<3$ (social problems present). A similar approach was taken by Curtis et al. ${ }^{28}$ in their analysis of neighborhood influences on a variety of health outcomes in a Canadian sample of children. Both cohesion and disorder were available every other data collection cycle starting at cycle 1 (children were 5 months of age) and therefore were analyzed as time-dependent variables (Table 1). Since values were missing for all children at cycle 8 (7 years), the observation at the 
TABLE 1 Description of considered explanatory variables in the QLSCD

\begin{tabular}{|c|c|c|}
\hline Variable & Description & Change over time \\
\hline \multicolumn{3}{|l|}{ Socio-economic/demographic } \\
\hline Sex & Male yes/no & Invariant \\
\hline Socioeconomic status (SES) ${ }^{a}$ & $\begin{array}{l}\text { Calculated based on gross household } \\
\text { income, and mother's and father's } \\
\text { education level, and job prestige; } \\
\text { categorized into tertiles-low, } \\
\text { middle, and high }\end{array}$ & Dependent \\
\hline Mother is an immigrant & Yes/no & Invariant \\
\hline Single parent family & Yes/no & Dependent \\
\hline \multicolumn{3}{|l|}{ Early life exposures } \\
\hline Rapid weight gain in infancy & $\begin{array}{l}\text { Highest two quintiles of average } \\
\text { monthly weight gain } \\
\text { from } 0 \text { to } 5 \text { months }\end{array}$ & Invariant \\
\hline $\begin{array}{l}\text { Mother smoked during } \\
\text { pregnancy }\end{array}$ & Yes/no & Invariant \\
\hline Breastfeeding status & $\begin{array}{l}\text { Exclusively breastfed to } 3 \text { months of age } \\
\text { or older; never breastfed; other }\end{array}$ & Invariant \\
\hline Birth weight ${ }^{b}$ & $\begin{array}{l}\text { Low }<2.5 \mathrm{~kg} ; \text { normal } \geq 2.5 \mathrm{~kg} \text { but } \leq 4 \mathrm{~kg} \\
\quad \text { high }>4 \mathrm{~kg}\end{array}$ & Invariant \\
\hline \multicolumn{3}{|l|}{ Genetic proxies ${ }^{c}$} \\
\hline Mother is obese & $\begin{array}{l}\text { Mother's BMI } \geq 30 \text { based on self-reported } \\
\text { height and weight }\end{array}$ & Invariant \\
\hline Overeating phenotype ${ }^{d}$ & $\begin{array}{l}\text { "Often" eats too much and/or "sometimes" } \\
\text { or "often" eats too fast }\end{array}$ & Invariant \\
\hline \multicolumn{3}{|c|}{ 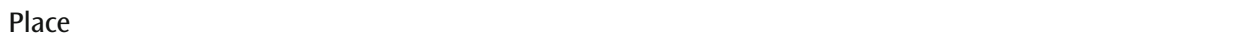 } \\
\hline Materially deprived & $\begin{array}{l}\text { Highest two quintiles of the material } \\
\text { deprivation factorial score }\end{array}$ & Invariant \\
\hline Socially deprived & $\begin{array}{l}\text { Highest two quintiles of the social } \\
\text { deprivation factorial score }\end{array}$ & Invariant \\
\hline High social cohesion & Scale score in the bottom $50 \%$ & Dependent \\
\hline High social disorder & Less than a perfect scale score $(<3)$ & Dependent \\
\hline Population density: & & Dependent \\
\hline High & $\begin{array}{l}\text { Census metropolitan areas with } \\
\quad \geq 100,000 \text { inhabitants }\end{array}$ & \\
\hline Medium & $\begin{array}{l}\text { Census agglomerations with } 10,000 \\
\text { to }<100,000 \text { inhabitants }\end{array}$ & \\
\hline Low & $\begin{array}{l}\text { Rural or small towns with }<10,000 \\
\text { inhabitants }\end{array}$ & \\
\hline
\end{tabular}

Time dependency reflects the fact that these variables were measured at all study time points $(4,6,7,8$, and 10 years of age) where all available data points were entered into the mixed models analysis. Values for social cohesion and disorder were not collected at 7 years for all children so the value at 6 years was used. For SES and population density, values were not collected at 4 years for all children so the value at 3.5 years (fourth data collection cycle) was used. All time invariant variables were measured the first data collection cycle of the original cohort study (5 months) except for the genetic proxies

${ }^{\mathrm{a}}$ For more information on how this variable was calculated and interpreted, please see Ref. ${ }^{25}$

${ }^{\mathrm{b}}$ Based on medical records at birth

${ }^{\mathrm{C}}$ Recognizing that these factors could also capture elements of the home environment, as well as lifestyle behaviors. Obesity status of the mother might also be considered an early life factor as this was measured when the child was 1.5 years (at the second data collection cycle)

${ }^{\mathrm{d}}$ Considered for inclusion based on research showing that at least half of the genetic influence on obesity operates through appetite (see Refs. ${ }^{26,27}$; measured at 4 years 
previous data collection cycle was carried forward (at 6 years) in order to be able to conduct the analysis.

Population Density The population density variable was constructed by ISQ by linking participants' postal codes to census data describing geographical areas using Statistics Canada's postal code conversion file. According to the linked census information, children were categorized as living in one of four types of geographical areas. $^{25}$ For the purpose of this study, these four categories were collapsed into three, namely, census metropolitan areas containing more than 100,000 inhabitants (high density), census agglomerations containing 10,000 to $<100,000$ inhabitants (medium density), or rural/small towns containing $<10,000$ inhabitants (low density). This variable was measured in all data collection cycles (except cycle 5 when children were 4 years of age) and, thus, was analyzed as a time-dependent variable. Because no children had population density collected at 4 years of age, the observation at the previous data collection cycle was carried forward (from 3.5 years).

\section{Other Explanatory Factors}

Other potentially important explanatory factors were identified from recent systematic reviews $^{29,30}$ and results of previous studies using the QLSCD. ${ }^{18,31,32}$ These variables were included in order to gain a more holistic understanding of weight change, as well as to control for potential confounding. A description of these variables is given in Table 1 . The mechanism by which explanatory variables could influence weight gain was not the primary focus here. Therefore, factors like lifestyle behaviors (e.g., physical activity, diet, and sleep), family functioning, parenting styles, food security, and general well-being of parents and child were not analyzed in this study as they were considered more proximate mediators.

\section{STATISTICAL ANALYSIS}

In order to achieve the study's main objective, a growth-curve or random-effects analysis was conducted using PROC MIXED in SAS, version 9.2, using the restricted maximum likelihood estimation method.

First, an exploratory unadjusted analysis was conducted to examine variable distributions and identify outliers and other potential problems with the data. Graphical analysis was conducted to investigate the shape of the BMI Z-score trend and to assess, in an exploratory fashion, the importance of the considered explanatory variables. Time was treated as a continuous variable (age in years) and was centered at the mean (approximately 7 years). To determine the base model from which to conduct further multivariable modeling, four "unadjusted" models, i.e., including only age as either linear, quadratic, cubic, or spline at 7 years, were compared using likelihood ratio tests. ${ }^{33}$ The $G$ matrix was assumed to be factor analytic. ${ }^{34}$ It was determined that the quadratic model fit the data better than the other models and was thus used in further model building. Modeling of explanatory factors involved adding all potential explanatory variables together to the unadjusted model and adding interactions between the explanatory variables and age and age $^{2}$. The interaction terms involving age and age ${ }^{2}$ were reduced by backwards elimination using $\alpha=0.05$.

The fit of the adjusted model was checked graphically to investigate violations of assumptions about random effects or the specification of fixed effects to identify potential outliers or observations having undue influence on the model and the need 
to transform particular covariates. Variance inflation factors were calculated for a cross-sectional model of BMI Z-score in order to assess multicollinearity between included explanatory variables. Ethics approval to conduct this analysis was given by the University of Ottawa Research Ethics Board-certificate number H 05-10-18.

\section{RESULTS}

Of the 2,120 cohort children, 1,799 had at least one BMI Z-score measure (out of five possible measures). Because of the cumulative effect of missing observations, 1,580 had complete data on all explanatory variables and could be included in the analysis (75\% of original sample). Almost $43 \%$ of included children had all five BMI Z-score response points, $24 \%$ had four, $12 \%$ had three, $9 \%$ had two, and $12 \%$ had one. The 540 children who were excluded were more likely to be from low socioeconomic status (SES) households, have immigrant mothers, live in materially and socially deprived neighborhoods, and exhibit the overeating phenotype compared to children who were included $\left(\chi^{2} P<0.05\right)$. Of the excluded children that had response measures, there were no differences in BMI Z-score between excluded and included children at any of the time points (ANOVA $F$ test).

Characteristics of included children are presented in Table 2. Both mean BMI and mean BMI-Z score increased over the study period (Table 3). The increasing BMI Zscore demonstrates that, on average, children were getting heavier for their height, age, and sex.

In the unadjusted BMI Z-score trend model (Table 4), the linear and quadratic parameters were positive and statistically significant, indicating that the growth in BMI among these children was accelerating from approximately 4-10 years of age.

The adjusted model is also presented in Table 4. Smoking during pregnancy and obesity status of the mother were significantly related to higher BMI Z-scores on average but did not interact with time. In terms of linear (or uniform) change over time, being male was associated with a faster rate of weight gain compared to being female, as was being from a low SES household relative to a high SES household. Living in a socially deprived neighborhood was significantly related to a slower rate of weight gain compared to nonsocially deprived areas. This association was also seen for living in medium density (census agglomerations) compared to high density areas (census metropolitan areas), between overeaters and nonovereaters, as well as between high birth weight relative to normal birth weight children. In terms of the nonlinear or nonconstant change component of the model (quadratic or age ${ }^{2}$ ), trends significantly differed for material deprivation, high social disorder, and rapid weight gain during infancy. Specifically, living in a materially deprived neighborhood was related to a higher accelerated weight gain relative to nonmaterially deprived areas. Conversely, living in a high disorder neighborhood was related to a lower accelerated weight gain relative to living in a nondisordered area, as was experiencing rapid weight gain during infancy versus normal growth. A visual example of the associations between significant place factors and weight gain over time is provided in Figure 2. Additionally, Figure 3 shows the differences in trends between levels of significant social and early life variables in the adjusted model.

Using a method developed by Lipsitz et al., ${ }^{35}$ the final model $R^{2}$ was calculated to be 0.80 , indicating good predictive ability. Graphical model checking did not reveal any major violations of statistical assumptions and indicated that the model adequately fit the data. Variance inflation factors did not indicate significant multicollinearity among explanatory factors. 
TABLE 2 Baseline descriptive data (4 years) of included children $(n=1,580)^{*}$

\begin{tabular}{|c|c|}
\hline Variable & Percentage $(n)$ \\
\hline \multicolumn{2}{|l|}{ Socio-economic/demographic } \\
\hline Male & $50.0(790)$ \\
\hline \multicolumn{2}{|l|}{ Socioeconomic status (SES) } \\
\hline Low & $31.9(499)$ \\
\hline Middle & $33.9(530)$ \\
\hline High & $34.2(535)$ \\
\hline Mother is an immigrant & $8.5(135)$ \\
\hline Single parent family & $13.2(208)$ \\
\hline \multicolumn{2}{|l|}{ Early life exposures } \\
\hline Rapid weight gain in infancy & $38.7(611)$ \\
\hline Mother smoked during pregnancy & $24.9(394)$ \\
\hline \multicolumn{2}{|l|}{ Breastfeeding status } \\
\hline$\geq 3$ months exclusively & $25.8(408)$ \\
\hline Never & $27.2(429)$ \\
\hline Other & $47.0(743)$ \\
\hline \multicolumn{2}{|l|}{ Birth weight } \\
\hline Low & $3.6(57)$ \\
\hline Normal & $85.7(1354)$ \\
\hline High & $10.7(169)$ \\
\hline \multicolumn{2}{|l|}{ Genetic proxies } \\
\hline Mother is obese & $9.9(157)$ \\
\hline Child overeats & $22.5(355)$ \\
\hline \multicolumn{2}{|l|}{ Place } \\
\hline Materially deprived & $37.9(599)$ \\
\hline Socially deprived & $37.1(586)$ \\
\hline High social cohesion & $47.8(732)$ \\
\hline High social disorder & $26.5(416)$ \\
\hline \multicolumn{2}{|l|}{ Population density } \\
\hline High & $66.4(1035)$ \\
\hline Medium & $11.2(174)$ \\
\hline Low & $22.5(350)$ \\
\hline
\end{tabular}

*Due to missing data on time-dependent variables, denominators for these variables are slightly less than 1,580

\section{DISCUSSION}

This study sought to examine the influence of place factors on children's weight status using longitudinal methods, while simultaneously controlling for social and early life factors, as well as genetic proxies. Significant associations were found for material and social deprivation, social disorder, and population density.

TABLE 3 Directly measured mean BMI and BMI Z-score by mean age for included children $(n=$ 1,580)

\begin{tabular}{llcc}
\hline Age (years) (SD) & BMI (SD) & BMI Z-score (SD) & Total N \\
\hline $4.2(0.26)$ & $15.7(1.60)$ & $0.014(1.23)$ & 1,352 \\
$6.1(0.25)$ & $15.7(1.90)$ & $0.031(1.15)$ & 1,008 \\
$7.1(0.25)$ & $16.1(2.27)$ & $0.043(1.10)$ & 1,296 \\
$8.1(0.26)$ & $16.8(2.59)$ & $0.170(1.10)$ & 1,161 \\
$10.1(0.26)$ & $18.4(3.24)$ & $0.342(0.99)$ & 1,123 \\
\hline
\end{tabular}

SD standard deviation 
TABLE 4 Unadjusted and adjusted BMI Z-score trend models: significant fixed effect parameters and their $95 \%$ confidence limits $(n=1,580)$

\begin{tabular}{|c|c|c|}
\hline Fixed effects & Parameter estimate & $95 \%$ Confidence limits \\
\hline \multicolumn{3}{|l|}{ Unadjusted model } \\
\hline Intercept & $0.062^{*}$ & $0.011,0.114$ \\
\hline Age & $0.049 * * *$ & $0.039,0.060$ \\
\hline $\mathrm{Age}^{2}$ & $0.012^{* * *}$ & $0.008,0.016$ \\
\hline \multicolumn{3}{|l|}{ Adjusted model } \\
\hline Intercept & $-0.136 *$ & $-0.268,-0.006$ \\
\hline \multicolumn{3}{|l|}{ Main effects } \\
\hline Age & $0.031 *$ & $0.006,0.055$ \\
\hline $\mathrm{Age}^{2}$ & $0.013^{* * *}$ & $0.007,0.020$ \\
\hline Male & $-0.116^{*}$ & $-0.213,-0.021$ \\
\hline Low SES ${ }^{\mathrm{a}}$ & $-0.123 * *$ & $-0.217,-0.033$ \\
\hline Middle SES ${ }^{\mathrm{a}}$ & $-0.087^{*}$ & $-0.163,-0.014$ \\
\hline Rapid weight gain in infancy & $0.333^{* * *}$ & $0.223,0.444$ \\
\hline Mother smoked during pregnancy & $0.135^{*}$ & $0.021,0.249$ \\
\hline High birth weight ${ }^{b}$ & $0.390 * * *$ & $0.236,0.549$ \\
\hline Low birth weight ${ }^{b}$ & $-0.456^{* *}$ & $-0.716,-0.197$ \\
\hline Mother is obese & $0.686^{* * *}$ & $0.528,0.845$ \\
\hline Child overeats & $0.422^{* * * *}$ & $0.308,0.537$ \\
\hline Materially deprived & 0.013 & $-0.097,0.124$ \\
\hline Socially deprived & 0.088 & $-0.011,0.188$ \\
\hline High social disorder & 0.043 & $-0.025,0.110$ \\
\hline Low population density ${ }^{c}$ & 0.011 & $-0.094,0.116$ \\
\hline Medium population density ${ }^{c}$ & 0.005 & $-0.123,0.135$ \\
\hline \multicolumn{3}{|l|}{ Linear age effects } \\
\hline Male & $0.047^{* * *}$ & $0.026,0.068$ \\
\hline Low SES ${ }^{a}$ & $0.050 * *$ & $0.022,0.078$ \\
\hline Middle SES ${ }^{a}$ & 0.020 & $-0.005,0.046$ \\
\hline Rapid weight gain in infancy & -0.009 & $-0.032,0.014$ \\
\hline Child overeats & $-0.026^{*}$ & $-0.052,-0.000$ \\
\hline High birth weight ${ }^{b}$ & $-0.043^{*}$ & $-0.076,-0.009$ \\
\hline Low birth weight ${ }^{b}$ & -0.001 & $-0.062,0.059$ \\
\hline Materially deprived & -0.004 & $-0.028,0.020$ \\
\hline Socially deprived & $-0.029^{*}$ & $-0.051,-0.007$ \\
\hline High social disorder & 0.020 & $-0.004,0.044$ \\
\hline Low population density ${ }^{c}$ & 0.002 & $-0.026,0.030$ \\
\hline Medium population density ${ }^{c}$ & $-0.050 * *$ & $-0.083,-0.016$ \\
\hline \multicolumn{3}{|l|}{ Quadratic age effects } \\
\hline Rapid weight gain in infancy & $-0.009 *$ & $-0.018,-0.001$ \\
\hline Materially deprived & $0.011^{* *}$ & $0.003,0.020$ \\
\hline High social disorder & $-0.012^{*}$ & $-0.022,-0.002$ \\
\hline
\end{tabular}

Model also adjusted for main effects of breastfeeding status, single parent family status, mother's immigrant status, and high social cohesion (all were not significant)

${ }^{*} P \leq 0.05 ;{ }^{* *} P \leq 0.01 ; * * * P \leq 0.0001$

${ }^{a}$ Reference is high SES

${ }^{\mathrm{b}}$ Reference is normal birth weight

${ }^{\mathrm{C}}$ Reference is high population density (census metropolitan area)

This study showed that weight change was positively related to age in this cohort of children, where rates of weight gain accelerated over time. The overall model suggests that early life factors play a role in childhood weight gain. For example, smoking during pregnancy and mother's obesity status were related to higher BMI Z- 

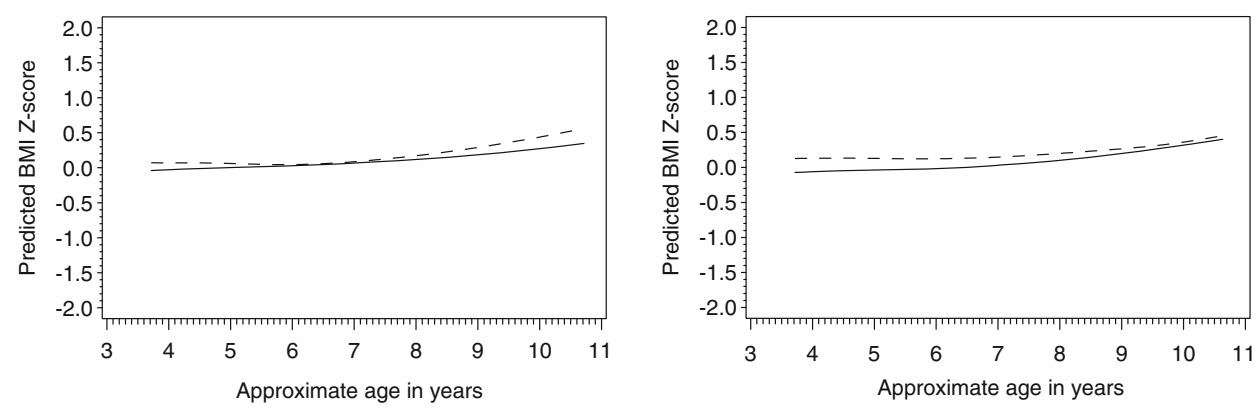

Material deprivation - Not materially deprived

Social deprivation _ Not socially deprived
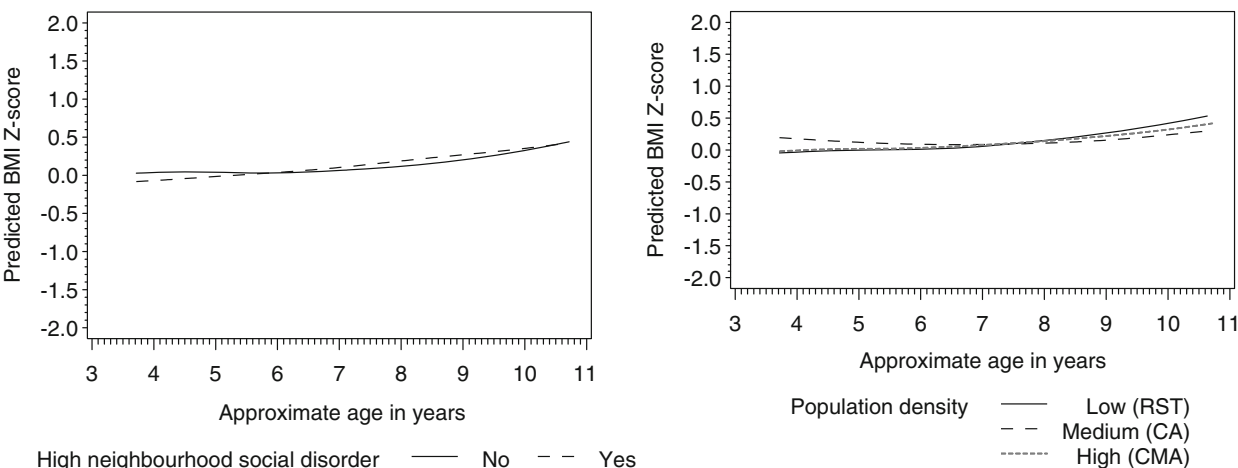

High neighbourhood social disorder — No - - Yes

FIGURE 2. Predicted BMI Z-score smoothed individual trends by significant place factors in the QLCSD, adjusted for other model covariates.

scores on average, and even though high birth weight and rapid weight gain in infancy were associated with slightly slower rates of weight change over time, children who had these characteristics had higher BMI Z-scores throughout the study compared to those without (Figure 3). Taken together, these results corroborate those of previous studies in a recent systematic review. ${ }^{29}$

In terms of individual and family characteristics, the difference in trends between males and females found in this study is in contrast to the findings of two other longitudinal studies that did not find significant interactions with time. ${ }^{11,36}$ Low SES being related to faster weight gain, on the other hand, has been demonstrated in other longitudinal studies. ${ }^{37}$

For the main explanatory factors of interest, living in materially deprived neighborhoods was related to higher accelerated weight gain. The general positive relationship uncovered here is in line with previous cross-sectional and longitudinal studies. ${ }^{9,38,39}$ Very few studies examining the effect of neighborhood characteristics on weight in children have actually partitioned neighborhood deprivation into two dimensions, such as was done in this study. Here, the influence of social deprivation was in contrast to that of material deprivation; the rate of weight gain among children who lived in areas with high social deprivation was slower than that of children who lived in nonsocially deprived areas. Using Québec information systems covering mortality, hospitalizations, and births, the creators of the deprivation index used in this study found that the two forms of deprivation had differing impacts on health. ${ }^{19}$ However, a cross-sectional study that used the same index did not find that social deprivation was significantly related to overweight among Canadian adults. ${ }^{22}$ 

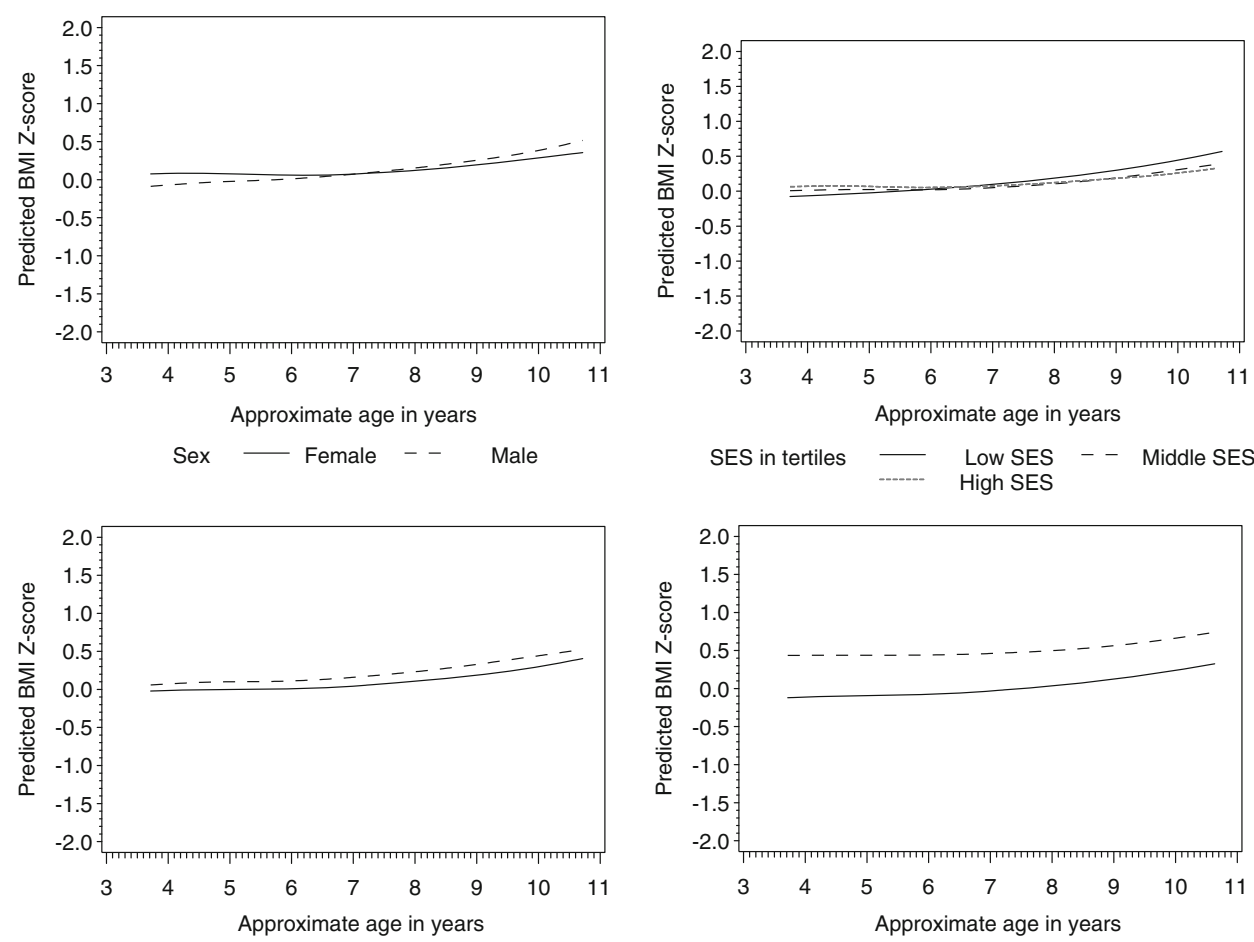

SES in tertiles — Low SES - - Middle SES High SES

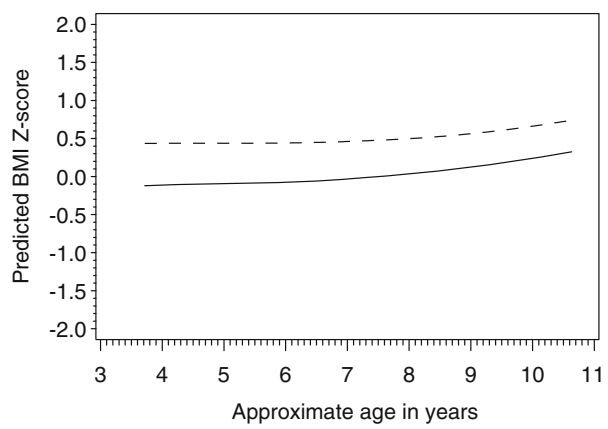

Mother smoked during pregnancy - No -- Yes

Child is an overeater - No -- Yes
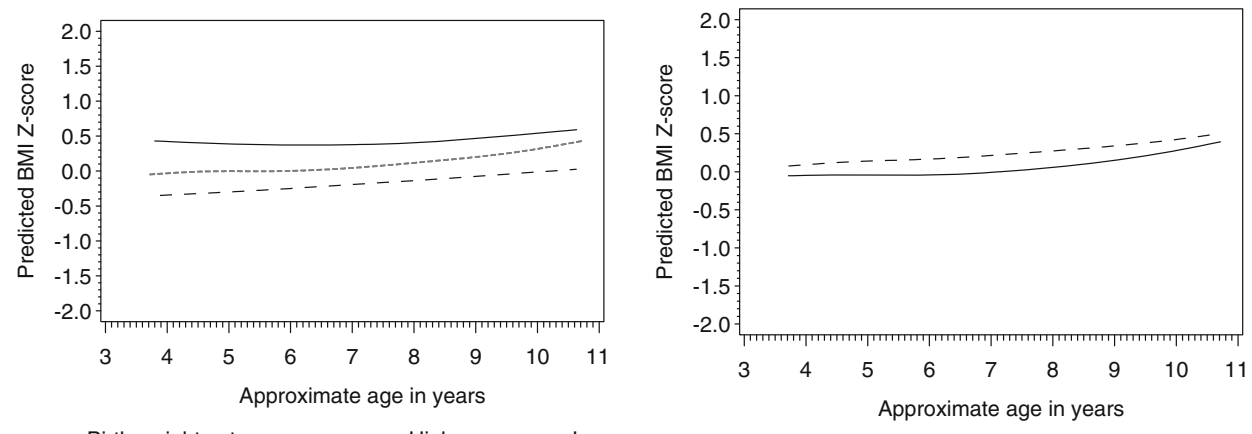

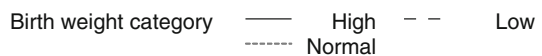

Rapid weight gain in infancy - No -- Yes

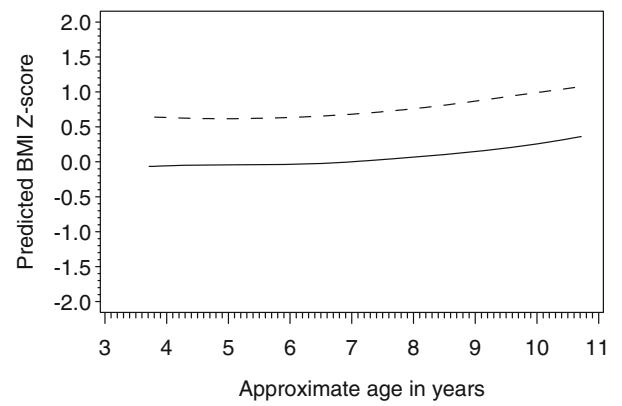

Obesity status of the mother _ Not obese

FIGURE 3. Predicted BMI Z-score smoothed individual trends by significant social and early life factors, adjusted for other model covariates. 
Similar to high social deprivation, living in neighborhoods with high social disorder was related to lower accelerated weight gain relative to not living in such areas. Again, few studies have related this neighborhood characteristic to weight status in children. Rather, studies have examined a similar concept, neighborhood safety, but have met with largely null results. ${ }^{9}$ Even though the findings for high social deprivation and high social disorder are in contrast to the original hypothesis, that they would be positively related to weight gain in children, they are interesting and informative nonetheless, and reflect the complexity of environmental influences on childhood weight status. One explanation for this finding is that such adverse social places may take longer to exert their weight promoting effects. While children are young, on the other hand, they may have the opposite effect-restricting growth, similar to what is often found in the early child development literature. ${ }^{28,40,41}$ On the other hand, it may be that these environmental influences provide protection from weight gain for reasons not yet understood.

Finally, population density was hypothesized to have a linear relationship with weight status, such that as density increased, weight gain would decrease. The findings here do not corroborate this: Children living in medium density (census agglomerations) areas exhibited slower growth than children in high density areas (census metropolitan areas), and there was no difference between low density (rural/ small towns) and high density areas. Overall, the literature in this area is methodologically heterogeneous with similarly mixed results. ${ }^{9,42}$ A study conducted by Statistics Canada used nationally representative data to determine the unadjusted regional distribution of child and adolescent overweight. They did not find that the prevalence of overweight significantly differed across census metropolitan areas, census agglomerations, and rural/small towns. ${ }^{43}$

The reason(s) for an inverse association between living in medium density areas and weight status may reflect a more complex reality than the original hypothesis was able to capture. For example, even though census agglomerations are less densely populated than census metropolitan areas, they have an urban core and can act like census metropolitan areas. ${ }^{44}$ Some have high functional metropolitan scores because they act as regional centers and therefore provide a range of services. ${ }^{44}$ Some may not have high functional scores but are located in close proximity to a census metropolitan area, benefiting from services provided close by. Thus, census agglomerations may function as more of a close-knit community than a census metropolitan area, with services close at hand, in contrast to rural/small towns whose residents must commute longer distances to access services and to go to work. The importance of population density on weight gain in children requires further study, and indeed longitudinal findings like these may not corroborate some earlier cross-sectional relationships observed. It is also possible that the relationship between population density and weight may change over time. This could also be said for the other factors considered here.

The findings of the present study should be interpreted in light of some limitations. First, this was a secondary analysis, which limited us to the variables that were collected. For example, material and social deprivation were measured once at the start of the cohort study and therefore could have changed over the study period. The place factors available in the QLSCD provided a 10,000 versus $100 \mathrm{ft}$ 
view of the place-weight status relationship, as more direct variables such as amenities, infrastructure, etc. were not available.

Due to the study design, some earlier time-dependent covariate data (from 5 months to 3.5 years) could not be accounted for in modeling. The study design also did not permit the use of sample weights. Therefore, the results are not necessarily generalizable to the Québec population. The overall model was fairly simplistic in regards to social-ecological theory. Other settings such as daycare and school were not included, and effect modification was not explored in order to keep the analysis manageable and parsimonious. Consideration should also be given to the importance of the place factors relative to the genetic proxies and factors operating in early life; variables such as obesity status of the mother, overeating, high birth weight, rapid weight gain in infancy, and smoking during pregnancy appear to be more strongly related to weight status than the individual sociodemographic/economic and place factors (see Figures 2 and 3).

Finally, children in this cohort may not follow a homogenous functional form of weight development, which can be characterized by a population-averaged model. This warrants a comparison between the results observed here and those derived from a group-based trajectory modeling approach, such as has been used in previous studies of childhood obesity. ${ }^{45-47}$

This study shows that, on average, cohort children were getting heavier over time and suggests the existence of individual and neighborhood social inequalities in childhood weight change. Further work is needed to clarify these relationships, especially for neighborhood-level factors. As in other studies, results here also point to early life as a potentially important developmental window for obesity. Future work, therefore, should also seek to determine the relationship between place characteristics during the perinatal period and these early life risk factors for obesity. Childhood obesity research is, by its very nature, complex. This merits a holistic approach where researchers should continue to focus "upstream" on the interrelationships between different contexts, settings, life stages, and generational transmission, in addition to traditional risk factors such as diet and physical activity.

\section{ACKNOWLEDGMENTS}

This work was supported by grants from the Canadian Institutes for Health Research. The QLSCD was conducted by Santé Québec, a division of the Institut de la Statistique du Québec (ISQ) and funded by the Ministry of Health and Social Services of Québec.

Disclaimer. This analysis was based on the ISQ QLSCD master files. All computations were prepared by the lead author. The responsibility for the use and interpretation of these data is solely that of the authors, not ISQ. The opinions expressed in this paper are those of the authors and do not represent the views of the ISQ.

OPEN ACCESS This article is distributed under the terms of the Creative Commons Attribution License which permits any use, distribution, and reproduction in any medium, provided the original author(s) and the source are credited. 


\title{
APPENDIX 1
}

TABLE 5 Neighborhood social scale items

\begin{tabular}{ll}
\hline Scale & Response categories
\end{tabular}

Social cohesion

Please tell me whether you strongly agree, agree, disagree, or strongly disagree about the following statement...

1. If there is a problem around here, the neighbors get together to deal with it Strongly agree $=1$

2. There are adults in the neighborhood that children can look up to Agree $=2$

3. People around here are willing to help their neighbors

Disagree $=3$

4. You can count on adults in this neighborhood to watch out that children are

Strongly disagree $=4$ safe and do not get in trouble

5. When I am away from home, I know that my neighbors will keep their eyes open for possible trouble

Social disorder

How much of a problem is the following in this neighbourhood...

1. Litter, broken glass or garbage?

2. Selling or using drugs?

3. Alcoholics and excessive drinking in public?

4. Groups of young people who cause trouble?

\author{
A big problem $=1$ \\ Somewhat of a \\ problem $=2$ \\ No problem $=3$
}

\section{REFERENCES}

1. Tremblay MS, Katzmarzyk PT, Willms JD. Temporal trends in overweight and obesity in Canada, 1981-1996. Int J Obes Relat Metab Disord. 2002; 26(4): 538-543.

2. Shields M. Overweight and obesity among children and youth. Health Rep. 2006; 17(3): 27-42.

3. Kohn M, Booth M. The worldwide epidemic of obesity in adolescents. Adolesc Med. 2003; 14(1): 1-9.

4. Lobstein T, Baur L, Uauy R. Obesity in children and young people: a crisis in public health. Obes Rev. 2004; 5(Suppl 1): 4-104.

5. Hertzman C. Framework for the social determinants of early child development. Encyclopedia on early child development 2010. http://www.enfant-encyclopedie.com/ pages/PDF/HertzmanANGxp.pdf. Accessed 1 Nov 2010.

6. Bernard P, Charafeddine R, Frohlich KL, Daniel M, Kestens Y, Potvin L. Health inequalities and place: a theoretical conception of neighbourhood. Soc Sci Med. 2007; 65 (9): 1839-1852.

7. Stafford M, Cummins S, Ellaway A, Sacker A, Wiggins R, Macintyre S. Pathways to obesity: identifying local, modifiable determinants of physical activity and diet. Soc Sci Med. 2007; 65(9): 1882-1897.

8. Black JL, Macinko J. Neighborhoods and obesity. Nutr Rev. 2008; 66(1): 2-20.

9. Carter MA, Dubois L. Neighbourhoods and child adiposity: a critical appraisal of the literature. Health Place. 2010; 16(3): 616-628.

10. Bell JF, Wilson JS, Liu GC. Neighborhood greenness and 2-year changes in body mass index of children and youth. Am J Prev Med. 2008; 35(6): 547-553.

11. Oliver LN, Hayes MV. Effects of neighbourhood income on reported body mass index: an eight year longitudinal study of Canadian children. BMC Public Health. 2008; 8: 16. 
12. Cecil-Karb R, Grogan-Kaylor A. Childhood body mass index in community context: neighborhood safety, television viewing, and growth trajectories of BMI. Health Soc Work. 2009; 34(3): 169-177.

13. Concepts, definitions and operational aspects: 5 month old enfants. Québec Longitudinal Study of Child Development (QLSCD 1998-2002). Institut de la statistique du Québec 2001, vol 1. no. 12. http://www.iamillbe.stat.gouv.qc.ca/bebe/bebe_n12_pdf_an.htm. Accessed 25 Oct 2010.

14. Survey description and methodology: 5-month old infants. Québec Longitudinal Study of Child Development (ELDEQ 1998-2002). Institut de la statistique du Québec 2000; vol 1, no. 1. http://www.iamillbe.stat.gouv.qc.ca/bebe/pdf/baby_no1-2.pdf. Accessed 26 Oct 2010.

15. Ogden CL, Kuczmarski RJ, Flegal KM, et al. Centers for disease control and prevention 2000 growth charts for the United States: improvements to the 1977 national center for health statistics version. Pediatrics. 2002; 109(1): 45-60.

16. Kuczmarski RJ, Ogden CL, Guo SS, et al. CDC growth charts for the United States: methods and development. Vital Health Stat. 2002; 11(246):1-190.

17. Must A, Anderson SE. Body mass index in children and adolescents: considerations for population-based applications. Int J Obes. 2006; 30(4): 590-594.

18. Desrosiers H, Dumitru V, Dubois L. Excess weight in children 4 to 7 years of age-targeting risk factors for intervention. Institut de la Statistique du Québec 2009. Vol. 4, No. 3.

19. Pampalon R, Raymond G. A deprivation index for health and welfare planning in Quebec. Chron Dis Can. 2000; 21(3): 104-113.

20. Pampalon R, Hamel D, Gamache P, Raymond G. A deprivation index for health planning in Canada. Chron Dis Can. 2009; 29(4): 178-191.

21. Pampalon R, Philibert M, Hamel D. Développement d'un système d'évaluation de la défavorisation des communautés locales et des clientèles de CLSC. Montréal, Québec: Institut national de santé publique du Québec; 2004. Report no. INSPQ-2004-024.

22. Lebel A, Pampalon R, Hamel D, Theriault M. The geography of overweight in Québec: a multilevel perspective. Can J Public Health. 2009; 100(1): 18-23.

23. Barnes-McGuire J. The reliability and validity of a questionnaire describing neighborhood characteristics relevant to families and young children living in urban areas. $J$ Community Psychol. 1997; 25(6): 551-566.

24. Statistics Canada. Microdata user guide: national longitudinal survey of children and youth—cycle 1. Ottawa: Human Resources Development Canada and Statistics Canada; 1997.

25. Variables dérivées de l'ÉLDEQ 1998-2008: partie A. Institut de la statistique du Québec 2009. http://www.jesuisjeserai.stat.gouv.qc.ca/pdf/doc_tech/E1aE11Variables_ Derivees1101.pdf. Accessed 25 Oct 2010.

26. Carnell S, Wardle J. Appetitive traits in children. New evidence for associations with weight and a common, obesity-associated genetic variant. Appetite. 2009; 53(2): 260263.

27. Llewellyn $\mathrm{CH}$, van Jaarsveld CH, Johnson L, Carnell S, Wardle J. Nature and nurture in infant appetite: analysis of the Gemini twin birth cohort. Am J Clin Nutr. 2010; 91(5): 1172-1179.

28. Curtis LJ, Dooley MD, Phipps SA. Child well-being and neighbourhood quality: evidence from the Canadian National Longitudinal Survey of Children and Youth. Soc Sci Med. 2004; 58(10): 1917-1927.

29. Monasta L, Batty GD, Cattaneo A et al. Early-life determinants of overweight and obesity: a review of systematic reviews. Obes Rev. 2010;11:695-708

30. Shrewsbury V, Wardle J. Socioeconomic status and adiposity in childhood: a systematic review of cross-sectional studies 1990-2005. Obesity. 2008; 16(2): 275-284.

31. Dubois L, Farmer A, Girard M, Peterson K, Tatone-Tokuda F. Problem eating behaviors related to social factors and body weight in preschool children: a longitudinal study. Int $J$ Behav Nutr Phys Act. 2007; 4: 9. 
32. Dubois L, Girard M. Early determinants of overweight at 4.5 years in a population-based longitudinal study. Int J Obes. 2006; 30(4): 610-617.

33. Cheng J, Edwards LJ, Maldonado-Molina MM, Komro KA, Muller KE. Real longitudinal data analysis for real people: building a good enough mixed model. Stat Med. 2010; 29(4): 504-520.

34. Fitzmaurice GM, Laird NM, Ware JH. Applied longitudinal analysis. Hoboken: Wiley; 2004.

35. Lipsitz SR, Leong T, Ibrahim J, Lipshultz S. A partial correlation coefficient and coefficient of determination for multivariate normal repeated measures data. Statistician. 2001; 50(1): 87-95.

36. Wardle J, Brodersen NH, Cole TJ, Jarvis MJ, Boniface DR. Development of adiposity in adolescence: five year longitudinal study of an ethnically and socioeconomically diverse sample of young people in Britain. BMJ. 2006; 332(7550): 1130-1135.

37. Hejazi S, Dahinten S, Ratner PA, Marshall SK. Developmental trajectories of weight status in childhood and adolescence. In: Bagchi D, editor. Global perspectives on childhood obesity. New York: Academic Press; 2011. pp. 21-29.

38. Oreskovic NM, Kuhlthau KA, Romm D, Perrin JM. Built environment and weight disparities among children in high- and low-income towns. Acad Pediatr. 2009; 9(5): 315-321.

39. Grow HM, Cook AJ, Arterburn DE, Saelens BE, Drewnowski A, Lozano P. Child obesity associated with social disadvantage of children's neighborhoods. Soc Sci Med. 2010; 71 (3): 584-591.

40. Kohen DE, Brooks-Gunn J, Leventhal T, Hertzman C. Neighborhood income and physical and social disorder in Canada: associations with young children's competencies. Child Dev. 2002; 73(6): 1844-1860.

41. Leventhal T, Brooks-Gunn J. The neighborhoods they live in: the effects of neighborhood residence on child and adolescent outcomes. Psychol Bull. 2000; 126(2): 309-337.

42. Dunton GF, Kaplan J, Wolch J, Jerrett M, Reynolds KD. Physical environmental correlates of childhood obesity: a systematic review. Obes Rev. 2009; 10(4): 393-402.

43. Shields M, Tjepkema M. Regional differences in obesity. Health Rep. 2006; 17(3): 61-67.

44. Mendelson R, Lefebvre J. Reviewing census metropolitan areas (CMA) and census agglomerations (CA) in Canada according to metropolitan functionality. Ottawa, Canada: Statistics Canada; 2003. Report no. 2003-001.

45. Hejazi S, Dahinten VS, Marshall SK, Ratner PA. Developmental pathways leading to obesity in childhood. Health Rep. 2009; 20(3): 63-69.

46. Li C, Goran MI, Kaur H, Nollen N, Ahluwalia JS. Developmental trajectories of overweight during childhood: role of early life factors. Obesity. 2007; 15(3): 760-771.

47. Mustillo S, Worthman C, Erkanli A, Keeler G, Angold A, Costello EJ. Obesity and psychiatric disorder: developmental trajectories. Pediatrics. 2003; 111(4 Pt 1): 851-859. 